\title{
Granulomatous lobular mastitis
}

\author{
Clinical update and case study
}

Meagan E Brennan, Melissa Morgan, Ghaith Bahjat Heilat, Kavitha Kanesalingam

\section{Background}

Granulomatous lobular mastitis (GM) is a rare chronic benign inflammatory breast condition. It can present a diagnostic challenge and mimic inflammatory carcinoma. It causes significant morbidity for affected patients.

\section{Objective}

The aim of this article is to provide a clinical update and case study for general practitioners, who are usually the first to see the patient.

\section{Discussion}

GM is usually idiopathic. The condition presents with a large painful lump with erythema. There is often ulceration, abscess and sinus formation. Triple assessment is needed to confirm diagnosis. GM may last for 12 months or more before healing occurs. Options for management are conservative/supportive care, oral prednisone or methotrexate, or surgical excision. At present, there is no universally accepted management strategy for GM; therefore, treatment will depend on the symptoms, extent of disease and patient preferences. Supporting the patient in coping with the long-term nature of GM and the chronic discharging lesions is essential.
GRANULOMATOUS LOBULAR MASTITIS (GM) is a chronic inflammatory breast condition that is often idiopathic. It is significant because it can mimic cancer, it is often difficult to diagnose and, despite it being a benign condition, its locally aggressive nature causes long-term pain and distress for affected patients. The aim of this article is to provide an update on the hypotheses of aetiology, diagnostic strategies and options for management. It also includes a patient's perspective.

\section{Aetiology}

GM is a condition characterised by non-caseating granulomas in the breast, often with abscess formation. These may be related to a foreign body, sarcoidosis, fungal infection or tuberculosis, but the process is often unexplained. Idiopathic GM was first described in 1971, ${ }^{1}$ and has since been described as an 'enigma'. ${ }^{2} \mathrm{GM}$ is rare, and incidence and prevalence have been difficult to estimate.

Patients with GM are typically women of childbearing age; a median age of approximately 35 years has been a relatively consistent finding. ${ }^{2-5}$ Hormonal factors are hypothesised to contribute because of the association with parity, current pregnancy and the oral contraceptive pill. GM is rarely seen in nulliparous women, and the majority of patients are within five years of a pregnancy, supporting the theory of a hormonal aetiology. ${ }^{3} \mathrm{GM}$ is usually a sterile process; however, an association with Corynebacterium species has been found in some studies. Corynebacterium kroppenstedtii has been found to create a distinct histopathological appearance termed cystic neutrophilic granulomatous mastitis. ${ }^{6,7} \mathrm{GM}$ is rare in women of Caucasian ethnicity. In the USA, it is more common in Hispanic and African American women when compared with women of other ethnicities. ${ }^{2,3}$ Personal or family history of breast cancer are not risk factors. GM has also been reported in males. ${ }^{3}$ An association between GM and hyperprolactinaemia levels has been found in cases of pituitary disease and craniopharyngioma. ${ }^{8}$

\section{Presentation and diagnosis}

GM is typically unilateral. The most common presenting symptom is a large tender lump, often several centimetres in diameter. ${ }^{2,3}$ Often this is associated with overlying skin changes of erythema and ulceration. Sinuses can develop, with discharge from the lesions. There are often multiple lumps and ulcers affecting one or more quadrants of the breast.

The differential diagnosis includes inflammatory breast cancer, locally advanced breast cancer with skin ulceration and other causes of breast inflammation such as non-lactational abscess and periductal mastitis. ${ }^{5,9,10}$ Fever is not usually present. Mammography typically shows non-specific features that are usually not diagnostic, such as asymmetric density. Ultrasonography is helpful when an abscess is present. This will show an irregular mass of mixed heterogenicity; however, this can also mimic cancer. ${ }^{2}$ The diagnosis of GM is made by core biopsy histopathology or fine-needle aspiration cytology. 
As it is an uncommon condition, GM is often unrecognised. Patients are prescribed repeated courses of antibiotics without response. Biopsy is often delayed unless there is an abscess requiring aspiration. The breast may be exquisitely tender, making imaging difficult. The long process before specialist consultation and recognition of the condition can lead to significant anxiety for the patient and the treating clinician.

\section{Management}

There is no consensus about the best management for GM. The options for management are:

- conservative/supportive care

- medication (prednisone and/or methotrexate)

- surgery.

Conservative management is advocated by many. If Corynebacterium species or other microbes are isolated, antibiotics should be prescribed according to sensitivities. ${ }^{6}$ Abscesses can be aspirated, and ulcerated areas dressed until spontaneous healing occurs. This usually takes 6-12 months or more, during which time more lumps and ulcers can develop and discharge. There is significant variation in the severity of the condition and the course of the illness. Perseverance is required, and the patient must be supported to accept the significant impact the condition can have on their lifestyle.

Prednisone and methotrexate have both been used for GM, with mixed results. Evidence comes from observational case series, and no randomised trials have been published. Because of the variability in the course of the disease, it is often difficult to know whether the illness has been shortened by these medications. Significant side effects can be experienced when these medications are prescribed for many months. Some studies have reported success with eight weeks of treatment. ${ }^{5}$ Recurrence on reduction or cessation of medications can be as high as $50 \%$.

Surgical excision can provide definitive diagnosis, especially when there is discordance with imaging and core biopsy. It may even be curative if the condition is extremely localised. However, GM frequently affects an entire quadrant of the breast or more, and excising the area is not a feasible option. It is also often difficult to determine the extent of disease to plan excision. Some authors recommend initial treatment with oral steroids or methotrexate, followed by surgical excision when the affected area has decreased in size. However, despite apparent curative surgical excision, a recurrence rate of up to $13 \%$ is reported. ${ }^{11}$

Appropriate treatment will be determined by the size and location of the lumps and skin lesions as well as the disease severity and patient preferences. Multidisciplinary care with input from a breast physician, surgeon, pathologist, radiologist and breast care nurse is ideal, with referral to an immunologist if methotrexate is considered. The education and support process is crucial, and the message for the patient that there is no 'quick fix' for this condition is essential. When the patient is adequately supported and has realistic expectations, they will likely adapt more successfully to the chronic nature of the condition. It is possible that the course of the illness will be shortened by medical management (eg steroids or methotrexate); however, this is not guaranteed, and significant side effects may be experienced. Regardless of the chosen management strategy, ongoing psychological support and wound management is required. The patient must also be warned that, regardless of treatment, there is likely to be significant scarring after healing.

\section{Conclusion}

GM is a rare chronic inflammatory breast condition. The aetiology is unknown. It is associated with childbearing age and parity. The diagnosis is frequently delayed as the condition is not recognised. The diagnosis can be made on percutaneous biopsy (core or fine-needle biopsy). Management options include conservative/supportive care, oral prednisone or methotrexate, or surgical excision. At present, there is no universally accepted management strategy for GM; therefore, treatment will depend on the symptoms, extent of disease and patient preferences. Supporting the patient in coping with the long-term nature of GM and the chronic discharging skin lesions is essential and requires input from a multidisciplinary team.

\section{Key points for general practice}

- GM is a rare inflammatory condition of the breast. It predominately occurs in premenopausal, parous women with a median age of approximately 35 years.

- GM can mimic cancer. At presentation, imaging and core biopsy are essential to exclude malignancy and confirm the diagnosis of GM.

- Management options include antibiotics (if Corynebacterium species are identified), aspiration of abscesses, oral steroids, methotrexate and surgery.

- Management requires a multidisciplinary breast consultation, taking into consideration the severity and patient preferences.

- Supportive care is a key part of management. Wound care and dressings may be required, and the patient requires support to cope with the chronic nature of the illness, which typically takes at least 6-12 months to resolve regardless of treatment.

\section{CASE}

Ms M, aged 36 years, presented with a tender lump adjacent to the nipple in the 9 o'clock position in her left breast. She had no history of breast problems and no family history of breast cancer. Ms M's background history included menarche at age 15 years and two pregnancies, six years and three years before presentation. Both pregnancies were complicated by gestational diabetes. She continued to have impaired glucose tolerance post-partum, managed with diet and exercise and regular endocrinology review. Ms M breastfed the babies for 15 months each and had no episodes of lactational mastitis. She commenced a contraceptive implant approximately 20 months post-partum and was taking no other medications. The remainder of her medical history was unremarkable. 
At presentation, Ms M described her breast lump as feeling 'like a bruise'. She was referred for mammography and breast ultrasonography, which showed no abnormality. Over the next couple of weeks, the discomfort reduced, but a firm lump several centimetres in size remained. Repeat ultrasonography showed a heterogeneous area of altered echotexture with non-specific, indeterminate features (Figure 1). A core biopsy of the breast showed non-specific inflammation and no signs of malignancy, and a fine-needle biopsy of a prominent axillary lymph node showed reactive features.

Ms M consulted several doctors, including a breast surgeon, and no clear diagnosis was made. The lump remained, and approximately six weeks after her initial presentation, another painful lump developed on lateral side of the same breast. Ultrasonography showed features of a breast abscess (Figure 2). The abscess was aspirated; neutrophils were seen on microscopy and no bacteria were grown on culture. Ms M was treated with several courses of antibiotics without improvement. The surgeon recommended open biopsy, incision and drainage, considering that the imaging and clinical features were discordant and inflammatory breast cancer remained a differential diagnosis.

Ms M sought a second surgical opinion at this time. There were now

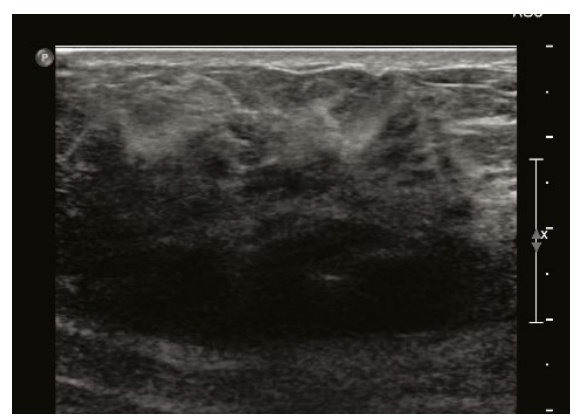

Figure 1. Ultrasound showing non-specific changes of altered echotexture and shadowing. This is typical in the early and the healing phases of granulomatous mastitis. significant signs of inflammation, with fever, severe pain and erythema. The second surgeon recommended against operative management and the surgery was cancelled. Repeat core biopsy was performed and this showed granulomatous inflammation and features of abscess; there was no growth on culture of aspirated fluid.

The diagnosis of GM was made. At this stage, Ms M was in significant pain and requiring the use of regular painkillers (ibuprofen and paracetamol). Breast abscesses were regularly forming; these discharged several weeks later. The abscesses left large areas of ulceration and some reduction in pain with spontaneous discharge (Figure 3). The ulceration and wounds remained open and active and did not heal despite being clean and sterile. Ms $\mathrm{M}$ was referred to an outpatient wound clinic at her local hospital for regular dressings. Painful abscesses continued to appear at a rate of one every few weeks for several more months. She was referred to an immunologist to discuss the benefits and risks of steroids or methotrexate, but she decided not to proceed with these medications as she was concerned about side effects and she understood that spontaneous healing would eventually occur. Five months after presentation there were signs of healing, but the scarring was significant (Figure 4).

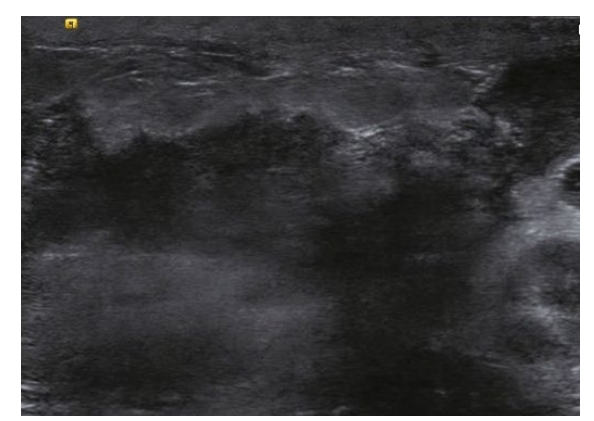

Figure 2. Ultrasound showing skin thickening and focal hypoechoic area suggesting abscess formation. This is typical in the active phases of granulomatous mastitis. Ultrasound-guided aspiration can be performed.
Ms M was distressed and exhausted by the experience of the diagnosis and management of her GM. She described feeling anxious in the first weeks as clinicians were unable to explain her symptoms. The possibility of inflammatory breast cancer was raised, further increasing concern. She was facing surgery but still had no diagnosis.

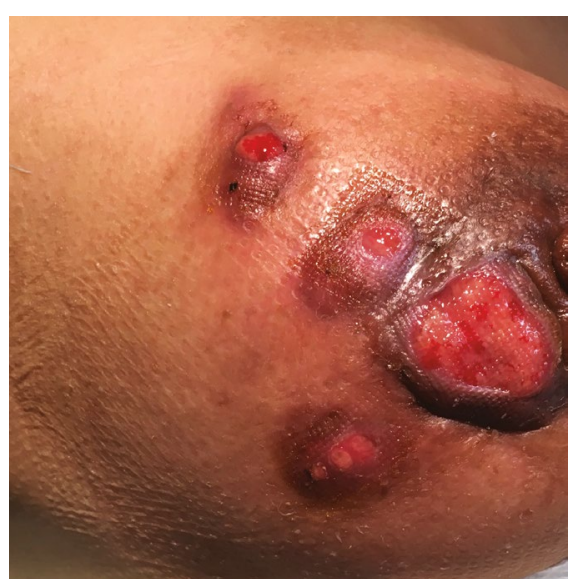

Figure 3. Ulceration and abscess formation typical of granulomatous mastitis

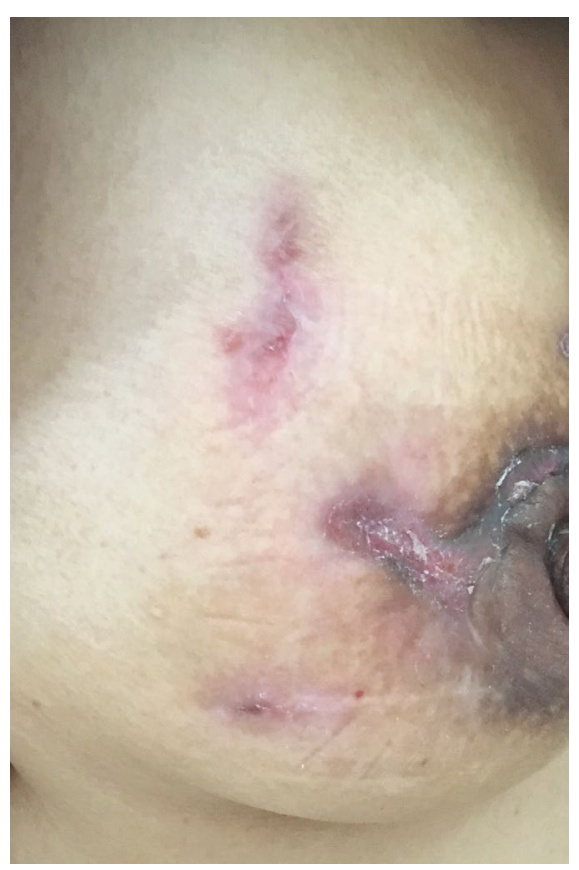

Figure 4. Healed areas of granulomatous mastitis. Significant scarring has resulted. 
She underwent several imaging studies and biopsies before the diagnosis was made. Even when granulomatous mastitis was diagnosed, its symptoms and likely clinical course were not explained well. The nurses managing her dressings provided good support but commented that they had not seen this condition before.

Ms M did her own research and found another doctor with experience treating the condition. She found a Facebook page that provided comfort, and she finally felt that she was not alone struggling with this rare disease. She also realised that she would never find a magic cure and that acceptance, dressings and a healthy lifestyle were the best things that she could do while she waited for gradual healing. She tried a variety of dietary supplements and exercise.

Ms $M$ has had symptoms for nine months. She says:

I wish I had gone online and found a support network earlier on. The biggest challenge for me was accepting the disease had no specific 'cure'; that there was no obvious medication to take. Once I realised this, I was able to focus on improving my lifestyle and mindset, which I think assisted in my gradual improvement.
Her advice to other women with this condition is:

Find a doctor who can support you through the process, accept that it takes time for this disease to resolve and seek support online from others who have been through it.

\section{Authors}

Meagan E Brennan FRACGP, FASBP, PhD, Senior Staff Specialist, Westmead Breast Cancer Institute, Westmead Hospital, NSW; Clinical Academic, School of Medicine Sydney, University of Notre Dame Australia, NSW; Clinical Associate Professor, Northern and Westmead Clinical Schools, School of

Medicine, Faculty of Medicine and Health, University of Sydney, NSW. meagan.brennan@sydney.edu.au

Melissa Morgan BSc (Hons), MCom, Patient Advocate, Westmead Breast Cancer Institute, Westmead Hospital, NSW

Ghaith Bahjat Heilat MD, JBGS, Fellow in Oncoplastic Surgery, Westmead Breast Cancer Institute, Westmead Hospital, NSW; Surgeon, Faculty of Medicine and Clinical Sciences, Yarmouk University, Jordan Kavitha Kanesalingam MBChB, FRCS, Fellow in Oncoplastic Surgery, Westmead Breast Cancer Institute, Westmead Hospital, NSW

Competing interests: None.

Funding: None.

Provenance and peer review: Not commissioned, externally peer reviewed.

\section{References}

1. Kessler E, Wolloch Y. Granulomatous mastitis: A lesion clinically simulating carcinoma. Am J Clin Pathol 1972;58(6):642-46.

2. Bashir MU, Ramcharan A, Alothman $S$, et al. The enigma of granulomatous mastitis: $A$ series. Breast Dis 2017;37(1):17-20. doi: 10.3233/ BD-160261.
3. Barreto DS, Sedgwick EL, Nagi CS, Benveniste AP. Granulomatous mastitis: Etiology, imaging, pathology, treatment, and clinical findings. Breast Cancer Res Treat 2018;171(3):527-34. doi: 10.1007/ s10549-018-4870-3.

4. Velidedeoglu M, Kilic F, Mete B, et al. Bilateral idiopathic granulomatous mastitis. Asian J Surg 2016;39(1):12-20. doi: 10.1016/j.asjsur.2015.02.003.

5. Gurleyik G, Aktekin A, Aker F, Karagulle H, Saglamc A. Medical and surgical treatment of idiopathic granulomatous lobular mastitis: A benign inflammatory disease mimicking invasive carcinoma. J Breast Cancer 2012;15(1):119-23. doi: 10.4048/jbc.2012.15.1.119.

6. Johnstone KJ, Robson J, Cherian SG, Wan Sai Cheong J, Kerr K, Bligh JF. Cystic neutrophilic granulomatous mastitis associated with Corynebacterium including Corynebacterium kroppenstedtii. Pathology 2017:49(4):405-412. doi: 10.1016/j.pathol.2017.01.006

7. Shoyele O, Vidhun R, Dodge J, et al. Cystic neutrophilic granulomatous mastitis: A clinicopathologic study of a distinct entity with supporting evidence of a role for Corynebacteriumtargeted therapy. Ann Diagn Pathol 2018;37:51-56. doi: 10.1016/j.anndiagpath.2018.08.005.

8. Nikolaev A, Blake CN, Carlson DL. Association between hyperprolactinemia and granulomatous mastitis. Breast J 2016;22(2):224-31. doi: 10.1111/ tbj.12552.

9. Chirappapha $P$, Thaweepworadej $P$, Supsamutchai C, Biadul N, Lertsithichai P. Idiopathic granulomatous mastitis: A retrospective cohort study between 44 patients with different treatment modalities. Ann Med Surg (Lond) 2018;36:162-67. doi: 10.1016/j.amsu.2018.11.001.

10. Azlina AF, Ariza Z, Arni T, Hisham AN. Chronic granulomatous mastitis: Diagnostic and therapeutic considerations. World J Surg 2003;27(5):515-18. doi: 10.1007/s00268-0036806-1.

11. Yilmaz TU, Gurel B, Güler SA, et al. Scoring idiopathic granulomatous mastitis: An effective system for predicting recurrence? Eur J Breast Health 2018:14(2):112-16. doi: 10.5152/ ejbh.2018.3709. 